\title{
Tensionless contact of a finite beam: Concentrated load inside and outside the contact zone
}

\author{
Yin Zhang · Kevin D. Murphy
}

Received: 28 February 2013 / Revised: 8 April 2013 / Accepted: 15 May 2013

CThe Chinese Society of Theoretical and Applied Mechanics and Springer-Verlag Berlin Heidelberg 2013

\begin{abstract}
For a finite beam with a nonzero gap distance, an asymmetric concentrated load can be either inside or outside of the contact zone. A new governing equation is given for the case of a concentrated load outside the contact zone. By numerically solving the left-side and right-side contact lengths of the beam, a criterion is established to determine whether the concentrated load is inside or outside the contact zone. A more general approach on the tensionless contact of a beam is thus presented.
\end{abstract}

Keywords Tensionless contact $\cdot$ Lift-off $\cdot$ Beam

\section{Introduction}

Tensionless contact, which is variably referred to as unbonded contact, unilateral, or receding contact $[1,2]$, is to describe the contact of a flexural structure, which may liftsoff, or say, separates from its contacting substrate due to the structural flexurality. The contact problem of a flexural structure is encountered in the indentation of such onedimensional nanostructures as nanobelt [1] and nanowire [2], or in the stiction of microcantilever [3,4]. An implicit assumption of the previous works on the tensionless contact of a beam or a plate is that the concentrated load is within

The project was supported by the National Natural Science Foundation of China (110212622 and 11023001) and Chinese Academy of Sciences (KJCX2-EW-L03).

\footnotetext{
Y. Zhang (西)

State Key Laboratory of Nonlinear Mechanics, Institute of Mechanics, Chinese Academy of Sciences, 100190 Beijing, China

e-mail: zhangyin@1nm.imech.ac.cn
}

K.D. Murphy

Department of Mechanical Engineering,

University of Connecticut,

Storrs, CT 06268, USA the contact zone $[1,2,5-8]$. For a finite $[1,2]$ or an infinite beam $[5,6]$ with zero gap distance from its contacting substrate, this assumption is always true. However, for a finite beam with a nonzero gap distance $[3,4,7]$, this assumption may not hold. For example, when a nanoindenter indents a micro-cantilever, which is modeled as a concentrated load applied to a beam, the concentrated load is shown to be outside the contact zone [4]. Because of lift-off, only a portion of beam is in contact with the substrate $[1,2,5$ 7]. When an asymmetric concentrated load acts on a beam with a nonzero gap distance, the location of the beam maximum displacement is in general different from that of the concentrated load. Physically, the contact zone is the neighborhood around the location of the beam maximum displacement. The concentrated load can thus be outside the contact zone. Two sets of the governing equations are presented for the scenarios of the concentrated load inside and outside the contact zone. A criterion is also given to tell which set of the governing equations should apply.

\section{Problem formulation}

Figure 1 is a schematic of a finite beam separated from a substrate with a nonzero gap distance of $Z_{\mathrm{o}}$. The coordinate system is also shown in Fig. 1. The beam is under a concentrated load of $P$. $E_{1}, L$ and $I$ are the beam Young's modulus, length and the area moment of inertia, respectively. $L_{1}$ and $L_{2}$ are the distances between $P$ and the left and right ends of the beam. Clearly, $L_{1}+L_{2}=L$. The beam deflection of $W$ as shown in Fig. 1 is divided into the following three parts

$W= \begin{cases}W_{1}, & \text { left-side lift-off region, } \\ W_{2}, & \text { contact region, } \\ W_{3}, & \text { right-side lift-off region. }\end{cases}$

When $P$ is inside the contact zone, the following governing equation set holds $[5,7]$

$E I \frac{\mathrm{d}^{4} W_{1}}{\mathrm{~d} X^{4}}=0, \quad W_{1}<Z_{\mathrm{o}}, \quad-L_{1}<X<X_{1}$, 
$E I \frac{\mathrm{d}^{4} W_{2}}{\mathrm{~d} X^{4}}+k\left(W_{2}-Z_{\mathrm{o}}\right)=P \delta(X)$,

$W_{2}>Z_{0}, \quad X_{1}<X<X_{2}$,

$E I \frac{\mathrm{d}^{4} W_{3}}{\mathrm{~d} X^{4}}=0, \quad W_{3}<Z_{\mathrm{o}}, \quad X_{2}<X<L_{2}$,

where $\delta(X)$ is the Dirac delta function. $X_{1}$ and $X_{2}$ are the points at which the beam separates from the substrate, as shown in Fig. 1. $k$ is the modulus of the Winkler elastic foundation to model the contacting substrate. For the contact between a beam with Young's modulus of $E_{1}$ and a substrate with Young's modulus of $E_{2}, k$ is given as follows [9]

$k=0.71 E_{2}\left(\frac{E_{2} B^{4}}{E_{1} I}\right)^{1 / 3}$.

Here $2 B$ is the beam width.

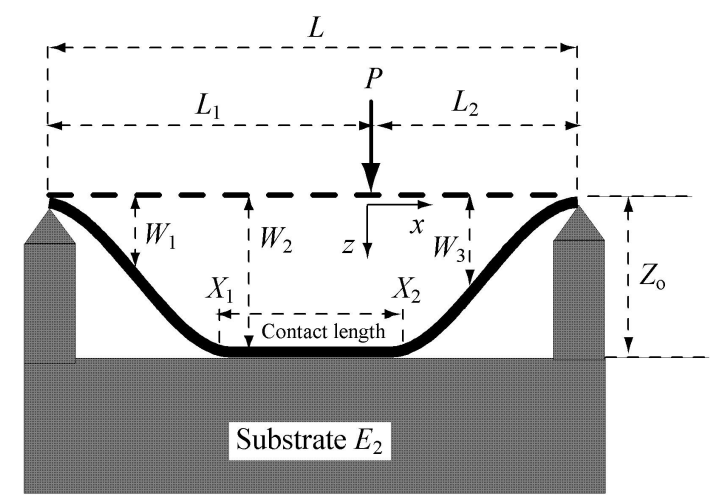

Fig. 1 Schematic diagram of a finite beam with a nonzero gap distance and the coordinate system

When $P$ is outside the contact zone, the following governing equation set holds

$E I \frac{\mathrm{d}^{4} W_{1}}{\mathrm{~d} X^{4}}=0, \quad W_{1}<Z_{\mathrm{o}}, \quad-L_{1}<X<X_{1}$,

$E I \frac{\mathrm{d}^{4} W_{2}}{\mathrm{~d} X^{4}}+k\left(W_{2}-Z_{\mathrm{o}}\right)=0, \quad W_{2}>Z_{\mathrm{o}}, \quad X_{1}<X<X_{2}$,

$E I \frac{\mathrm{d}^{4} W_{3}}{\mathrm{~d} X^{4}}=P \delta(X), \quad W_{3}<Z_{\mathrm{o}}, \quad X_{2}<X<L_{2}$.

Here $L_{1} \geqslant L_{2}$ is assumed and $P$ thus locates in the right-side lift-off zone of $W_{3}$. If $L_{1} \leqslant L_{2}, P$ will be in the left-side liftoff zone of $W_{1}$. Here only the case of $L_{1} \geqslant L_{2}$ is computed.

The following nondimensionalization scheme is introduced for computation comparison $[5,7]$

$$
\begin{array}{lll}
\xi_{1}=\beta X_{1}, & \xi_{2}=\beta X_{2}, & l_{1}=\beta L_{1}, \\
l_{2}=\beta L_{2}, & l=\beta L, & z_{\mathrm{o}}=\beta Z_{\mathrm{o}}, \\
w=\beta W, & \xi=\beta X, & F=\frac{P}{4 \beta^{2} E I},
\end{array}
$$

where $\beta$ is a parameter defined as follows

$\beta^{4}=\frac{k}{4 E_{1} I}$ with the unit of $\mathrm{m}^{-1}$. Physically, $\beta^{-1}$ is also the length used to evaluate the effect of beam bending on the contact [10].

Equation (2) is now nondimensionalized as

$$
\begin{aligned}
& \frac{\mathrm{d}^{4} w_{1}}{\mathrm{~d} \xi^{4}}=0, \quad w_{1}<z_{0}, \quad-l_{1}<\xi<\xi_{1}, \\
& \frac{\mathrm{d}^{4} w_{2}}{4 d \xi^{4}}+w_{2}-z_{\mathrm{o}}=F \delta(\xi), \quad w_{2}>z_{0}, \quad \xi_{1}<\xi<\xi_{2}, \\
& \frac{\mathrm{d}^{4} w_{3}}{\mathrm{~d} \xi^{4}}=0, \quad w_{3}<z_{\mathrm{o}}, \quad \xi_{2}<\xi<l_{2} .
\end{aligned}
$$

Equation (4) is now nondimensionalized as

$$
\begin{aligned}
& \frac{\mathrm{d}^{4} w_{1}}{\mathrm{~d} \xi^{4}}=0, \quad w_{1}<z_{0}, \quad-l_{1}<\xi<\xi_{1}, \\
& \frac{\mathrm{d}^{4} w_{2}}{4 \mathrm{~d} \xi^{4}}+w_{2}-z_{\mathrm{o}}=0, \quad w_{2}>z_{0}, \quad \xi_{1}<\xi<\xi_{2}, \\
& \frac{\mathrm{d}^{4} w_{3}}{\mathrm{~d} \xi^{4}}=4 F \delta(\xi), \quad w_{3}<z_{\mathrm{o}}, \quad \xi_{2}<\xi<l_{2} .
\end{aligned}
$$

The solutions to Eq. (7) are given as follows [7]

$$
w_{1}=A_{1} \xi^{3}+B_{1} \xi^{2}+C_{1} \xi+D_{1},
$$

$w_{2}=A_{2} \cosh \xi \sin \xi+B_{2} \cosh \xi \cos \xi+C_{2} \sinh \xi \sin \xi$

$$
\begin{aligned}
& +D_{2} \sinh \xi \cos \xi-\frac{F}{2} \sinh |\xi| \\
& +\frac{F}{2} \cosh \xi \sin |\xi|+z_{0}, \\
w_{3}= & A_{3} \xi^{3}+B_{3} \xi^{2}+C_{3} \xi+D_{3} .
\end{aligned}
$$

The difficulty of deriving the solutions to Eqs. (7) and (8) is on the particular part. Weitsman's approach [5] of constructing a particular solution for Eq. (7) also applies to Eq. (8). For brevity, the solutions to Eq. (8) are directly given as follows

$$
\begin{aligned}
w_{1}= & a_{1} \xi^{3}+b_{1} \xi^{2}+c_{1} \xi+d_{1}, \\
w_{2}= & a_{2} \cosh \xi \sin \xi+b_{2} \cosh \xi \cos \xi \\
& +c_{2} \sinh \xi \sin \xi+d_{2} \sinh \xi \cos \xi+z_{0}, \\
w_{3}= & a_{3} \xi^{3}+b_{3} \xi^{2}+c_{3} \xi+d_{3}+4 F H_{3}(\xi),
\end{aligned}
$$

where $A_{i}, B_{i}, C_{i}, D_{i}$ and $a_{i}, b_{i}, c_{i}, d_{i}(i=1,2,3)$ in Eqs. (9) and (10) are the unknown constants to be determined. $H_{3}$ is a function defined as

$$
\begin{aligned}
H_{3}(\xi) & =\int_{-l_{1}}^{l_{2}} \int_{-l_{1}}^{l_{2}} \int_{-l_{1}}^{l_{2}} \int_{-l_{1}}^{l_{2}} \delta(\xi) \mathrm{d} \xi \mathrm{d} \xi \mathrm{d} \xi \mathrm{d} \xi \\
& = \begin{cases}0, & \xi \leqslant 0, \\
\frac{\xi^{3}}{6}, & \xi>0 .\end{cases}
\end{aligned}
$$

The matching conditions at $\xi=\xi_{1}, \xi_{2}$ can be derived by a variation approach [11] as follows 


$$
\begin{array}{ll}
w_{1}\left(\xi_{1}\right)=w_{2}\left(\xi_{1}\right), & \frac{\mathrm{d} w_{1}}{\mathrm{~d} \xi}\left(\xi_{1}\right)=\frac{\mathrm{d} w_{2}}{\mathrm{~d} \xi}\left(\xi_{1}\right), \\
\frac{\mathrm{d}^{2} w_{1}}{\mathrm{~d} \xi^{2}}\left(\xi_{1}\right)=\frac{\mathrm{d}^{2} w_{2}}{\mathrm{~d} \xi^{2}}\left(\xi_{1}\right) & \frac{\mathrm{d}^{3} w_{1}}{\mathrm{~d} \xi^{3}}\left(\xi_{1}\right)=\frac{\mathrm{d}^{3} w_{2}}{\mathrm{~d} \xi^{3}}\left(\xi_{1}\right), \\
w_{2}\left(\xi_{2}\right)=w_{3}\left(\xi_{2}\right), & \frac{\mathrm{d} w_{1}}{\mathrm{~d} \xi}\left(\xi_{2}\right)=\frac{\mathrm{d} w_{3}}{\mathrm{~d} \xi}\left(\xi_{2}\right), \\
\frac{\mathrm{d}^{2} w_{2}}{\mathrm{~d} \xi^{2}}\left(\xi_{2}\right)=\frac{\mathrm{d}^{2} w_{3}}{\mathrm{~d} \xi^{2}}\left(\xi_{2}\right), & \frac{\mathrm{d}^{3} w_{2}}{\mathrm{~d} \xi^{3}}\left(\xi_{2}\right)=\frac{\mathrm{d}^{3} w_{3}}{\mathrm{~d} \xi^{3}}\left(\xi_{2}\right) .
\end{array}
$$

The above matching conditions in essence indicate the continuity of the displacement, slope, moment and shear force at the separation points of $\xi_{1}$ and $\xi_{2}$ [2]. The matching conditions are also frequently referred to as the transversality conditions $[11,12]$.

Two constraint conditions, which indicates the beam displacement at the separation points, are given as follows [7]

$w_{2}\left(\xi_{1}\right)=z_{0}, \quad w_{2}\left(\xi_{2}\right)=z_{0}$.

Here the capillary [13] and the adhesion [14] effects, which play a very important role in the micro/nanostructures, are not considered. Once these effects are considered, the above constraint conditions change correspondingly [14].

For a hinged-hinged beam, the boundary conditions are as follows

$$
\begin{array}{ll}
w_{1}\left(-l_{1}\right)=0, & \frac{\mathrm{d}^{2} w_{1}}{\mathrm{~d} \xi^{2}}\left(-l_{1}\right)=0, \\
w_{3}\left(l_{2}\right)=0, & \frac{\mathrm{d}^{2} w_{3}}{\mathrm{~d} \xi^{2}}\left(l_{2}\right)=0 .
\end{array}
$$

There are fourteen unknown constants to be determined either for the case of the concentrated load inside contact zone $\left(A_{i}, B_{i}, C_{i}, D_{i}(i=1,2,3), \xi_{1}\right.$ and $\left.\xi_{2}\right)$ or for the case of the load outside the contact zone $\left(a_{i}, b_{i}, c_{i}, d_{i}(i=1,2,3), \xi_{1}\right.$ and $\left.\xi_{2}\right)$. The transversality, constraint and boundary conditions of Eqs. (12), (13), and (14) give fourteen equations in total to solve the fourteen unknowns. Because of the unknown property of the contact zone (i.e., $\xi_{1}$ and $\xi_{2}$ ), solving these fourteen unknowns is a nonlinear problem and the Newton-Rhapson method is used [7]. For the NewtonRhapson method to start, the fourteen unknowns need to be guessed first. The computation is not very sensitive to the initial guesses and converges after a few iterations.

\section{Results and discussion}

As shown in Fig. 1, the concentrated load is at the origin of the coordinate system. In this study the concentrated load starts at the center of the beam and then moves towards the beam right end. When the concentrated load is at the center of a hinged-hinged beam, if the beam is in contact with the substrate, the concentrated load must be inside the contact zone because the loading location is also the location of the maximum beam displacement. If the concentrated load moves rightward and out of the contact zone, the concen- trated load can only be on the right-side lift-off zone as assumed by Eq. (8). When the concentrated load is inside the contact zone, we have $\xi_{1}<0$ and $\xi_{2}>0$; when the concentrated load is outside the contact zone, we have $\xi_{1}<0$ and $\xi_{2}<0$ for the scenario of the load moving rightward. Therefore, the criterion here to tell whether the concentrated load locates inside or outside the contact zone is $\xi_{2}=0$. Clearly, if $F$ moves leftward, the criterion will be $\xi_{1}=0$.

Here an example with the parameters of $l=4, z_{0}=0.6$ and $F=0.2$ is presented. Figure 2a shows the variation of total contact length as $l_{1}$ increases (or say, $F$ moves rightward) from the center of $l_{1}=2$; Figure $2 \mathrm{~b}$ shows the variation of the leftside and rightside contact lengths as $l_{1}$ increases. When $F$ is inside the contact zone, the rightside contact length is $\xi_{2}$ and the leftside contact length is $-\xi_{1}$; the total contact length here is calculated as $\xi_{2}-\xi_{1}$. As seen in Fig. 2a, when the load moves rightward, the total length increases and then reduces. It needs to point out that the concentrated load at the beam center does not necessarily give the largest total contact length, which is discussed in detail in Ref. [7]. In contrast, the right-side/leftside contact length decreases/increases monotonically as $l_{1}$ increases. The rightside contact length of $\xi_{2}$ becomes zero when $l_{1} \approx 0.692 l=2.768$. $F$ will be outside the contact zone if it moves further rightward. Figure 3 shows the two beam deflection shapes at $l_{1}=0.5 l$ and $l_{1}=0.69 l$, respectively. Both are the scenario of the concentrated load inside the contact zone. For the symmetric loading of $l_{1}=0.5 l$, $-\xi_{1}=\xi_{2}=0.6172$; for the asymmetric loading of $l_{1}=0.69 l$, $\xi_{1}=-1.0798$ and $\xi_{2}=0.000303$.

When $l_{1}>0.692 l$, the concentrated load is outside the contact zone; Equation (7) becomes invalid and Eq. (8) applies. Figure 4 shows the two cases of $F$ outside the contact zone with $l_{1}=0.7 l$ and $l_{1}=0.75 l$, respectively. In Fig. $4 \mathrm{a}$ of $l_{1}=0.7 l, \xi_{1}=-1.105$ and $\xi_{2}=-0.04778$; In Fig. $4 \mathrm{~b}$ of $l_{1}=0.75 l, \xi_{1}=-1.225$, and $\xi_{2}=-0.276$. The $l_{1}$ difference between Fig. $3 \mathrm{~b}$ and Fig. $4 \mathrm{a}$ is small and it is found that their differences in the beam deflection shapes and contact lengths are also small. The beam is an Euler-Bernoulli beam and the
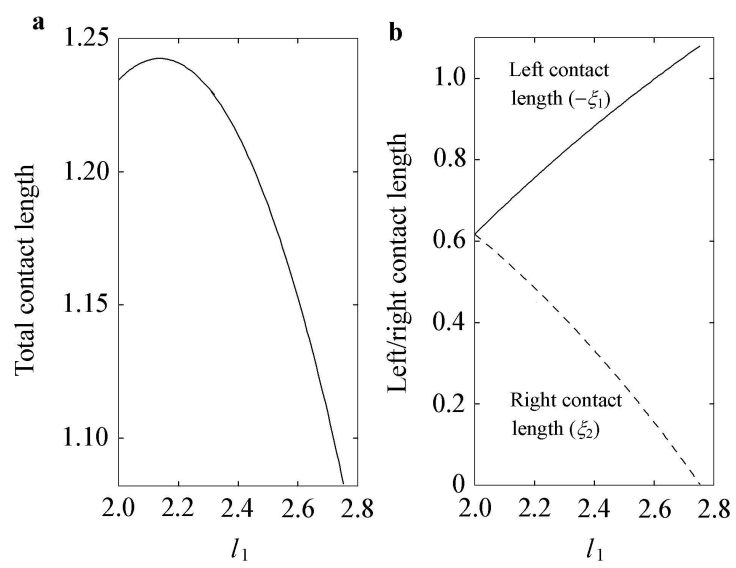

Fig. 2 a The total contact length versus $l_{1}$; b The leftside/rightside contact length versus $l_{1}$ 

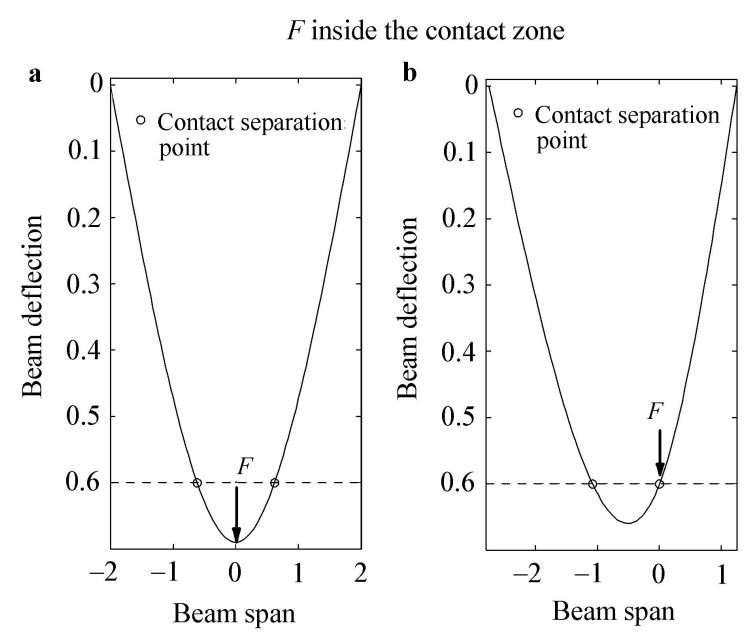

\section{Summary}

An asymmetric concentrated load can be either inside or outside the contatc zone of a flexural beam with a nonzero gap distance. Two sets of governing equations are needed for these two scenarios. A criterion is established to tell whether the concentrated load is inside or outside the contact zone. As the concentrated load moves out of the contact zone, the changes of both the beam deflection shape and the contact length are shown to be smooth.

\section{References}

1 Zhang, Y.: Extracting nanobelt mechanical properties from nanoindentation. J. Appl. Phys. 107, 123518 (2010)

Fig. 3 Beam deflection shapes with the concentrated load inside the contact zone. a $l_{1}=0.50 l ; \mathbf{b} l_{1}=0.69 l$
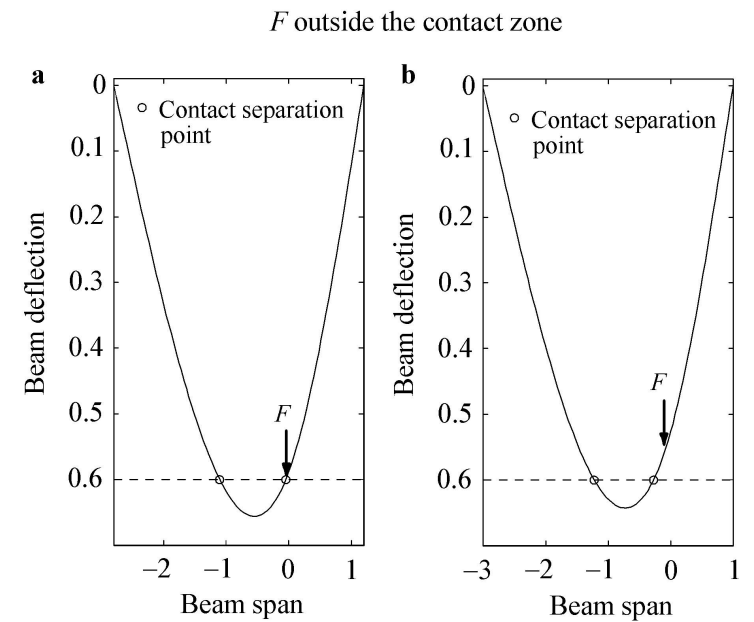

Fig. 4 Beam deflection shapes with the concentrated load outside the contact zone. a $l_{1}=0.70 l ; \mathbf{b} l_{1}=0.75 l$

foundation is a linear Winkler foundation. The nonlinearity of the tensionless contact is caused by the unknown feature of the contact zone. Mathematically, the fact that the concentrated load moves out of the contatc zone corresponds to the switching of the governing equations from Eq. (7) to Eq. (8), which introduces another nonlinearity and this nonlinearity does not cause any abrupt change.

2 Zhang, Y., Zhao Y.P.: Modeling nanowire indentation test with adhesion effect. J. Appl. Mech. 78, 011007 (2011)

3 Mastrangelo, C.H., Hsu, C.H.: Mechanical stability and adhesion of microstructures under capillary forces - part I: Basic theory. J. Microelectromech. Syst. 2, 33-43 (1993)

4 Jones, E.E, Begley, M.R., Murphy, K.D.: Adhesion of microcantilever subjected to mechanical point loading: Modeling and experiments. J. Mech. Phys. Solids 51, 1601-1622 (2003)

5 Weitsman, Y.: On Foundations that reacts in compression only. J. Appl. Mech. 37, 1019-1030 (1970)

6 Weitsman, Y.: A tensionless contact between a beam and an elastic half-space. Int. J. Engr. Sci. 10, 73-81 (1972)

7 Zhang, Y., Murphy, K.D.: Response of a finite beam in contact with a tensionless foundation under symmetric and asymmetric loading. Int. J. Solids Struct. 41, 6745-6758 (2004)

8 Zhang, Y., Murphy, K.D.: Tensionless contact of a finite circular plate. Acta Mech. Sin. 28, 1374-1381 (2012)

9 Biot, M.A.: Bending of an infinite beam on an elastic foundation. J. Appl. Mech. 4, 1-7 (1937)

10 Castillo, J., Barber, J.S.: Lateral contact of slender prismatic bodies. Proc. R. Soc. London, Ser. A 453, 2397-2412 (1997)

11 Kerr, A.D.: On the derivation of well posed boundary value problems in structural mechanics. Int. J. Solids Struct. 12, 1-11 (1976)

12 Liu, J.L.: Theoretical analysis on capillary adhesion of microsized plates with a substrate. Acta Mech. Sin. 26, 217-223 (2010)

13 Liu, J.L., Feng, X.Q.: On elastocapillarity: A review. Acta Mech. Sin. 28, 928-940 (2012)

14 Zhang, Y., Zhao, Y.P.: Flexural contact in MEMS stiction. Int. J. Solids Struct. 49, 2203-2214 (2012) 